\title{
Tracking Metastable Phase Selection during Devitrification in a Metallic Glass
}

\author{
Lin Zhou ${ }^{1 *}$, Fanqiang Meng ${ }^{1}$, Shihuai Zhou ${ }^{1}$, Kewei Sun ${ }^{1}$, Tae-Hoon Kim ${ }^{1}$, Ryan Ott ${ }^{1}$, Ralph \\ Napolitano $^{1}$, Matthew J. Kramer ${ }^{1}$ \\ 1. Ames Laboratory, Ames, IA, USA. \\ * Corresponding author: linzhou@ameslab.gov
}

Devitrification of metallic glasses can lead to a wide range of metastable phases, many not accessible during solidification from the melt. Advanced in situ characterization capabilities now provide a window for real-time observation of phase transition down to atomic level. In this study, we measured competitive nucleation and growth involving metastable phases crystallizing from an $\mathrm{Al}_{90} \mathrm{Sm}_{10}$ glass. The growth of a large unit cell crystalline phase $\left(\mathrm{Al}_{120} \mathrm{Sm}_{22}\right.$, $\varepsilon$-phase $)$ is accompanied by intermittent formation of Al-clusters at the growth front, modifying the crystal-glass interface movement and promoting a "cauliflower" microstructure. Subsequent solid-solid phase transformations showed a complex eutectic growth of three phases. The obtained structural and dynamic information provides valuable insight into metastable phase selection when coupled with atomic scale modeling. Challenges and opportunities for such detailed studies will be discussed [1-2].

Amorphous ribbon was fabricated using melt spinning process with a tangential wheel speed of $30 \mathrm{~m} / \mathrm{s}$. Time-resolved synchrotron X-ray scattering was performed at the Advanced Photon Source (APS) at Argonne National Laboratory. TEM sample was prepared by lift-out using an FEI HELIOS focus ion beam from a relative uniform region (away from gas pocket) in the wheel side of a melt-spun ribbon. In situ heating was achieved using an FEI NanoExTM-i/v TEM holder. TEM characterization was performed on a probe aberration corrected Titan Themis equipped with a Super-X EDS detector.

Figure 1 shows sequential HAADF STEM images taken after $\sim 9 \mathrm{~s}$ of heating steps at the targeted $175^{\circ} \mathrm{C}$. It appears that the $\varepsilon$-phase cannot fully accommodate the $\mathrm{Al}$ content of the glass. The $\varepsilon$ crystal is surrounded by a boundary with darker contrast in the HAADF image, indicative of an Al-rich region. As the $\varepsilon$-phase grows, more Al is concentrated at the glass/crystalline boundary. Finally, the concentration is sufficient to form lenticular nanocrystallites of $f c c$-Al. Some examples of the Al nanocrystallites are indicated by white arrows in Fig. 1. Subsequent $\varepsilon$-phase growth can only proceed from regions where there are no Al nanocrystallites (indicated by red arrows). The growth front is quite irregular, with faster growing regions bulging out, only to form reentrants, which then reconnect and encapsulating $f c c-\mathrm{Al}$ nanocrystals. The Al nanocrystallites grow much slower than $\varepsilon$-phase, likely through diffusion of $\mathrm{Al}$ from the nearby boundary [2].

Figure 2 shows high-resolution ex-situ HRSTEM (HRSTEM) (top row) and SAED of the [100], [111] and [110] zone axis of the $\varepsilon$-phase. The SAED's regions were large enough to include an average phase fraction of the intracrystalline $f c c-\mathrm{Al}$. The contrast variation in $\mathrm{Sm}$ sites in (a)-(c) is caused by $\mathrm{Sm}$ occupancy difference in the two atomic sites along the electron beam direction. Arcs appearing close to the $\{110\}$ and $\{100\}$ diffraction spots, as indicated by white arrows are from the slightly misoriented fcc-Al indicative of a near coincident lattice. In particular, the [011] diffraction pattern shows a very close match of the d-spacing of the $\{4,3,3\}_{\varepsilon}$ and the $\{1,1,1\}_{f c-A l}$. The arc of the $\{111\}_{f c c-A l}$ (as indicated by white arrow) from the $\{4,3,3\}$ toward the $\{2,4,4\}$ and the symmetrical arc of the $f c c-A l\{002\}$ (as 
indicated by yellow arrow, bottom) defines the relationship between the $\varepsilon$ and $f c c$-Al follows a simple relationship where their $\{$ h00 $\}$ faces, and $<00 \mathrm{k}>$ directions align within $\sim 7^{\circ}[2,3]$.

\section{References:}

[1] M.B. Kruger et al., Science 249 (1990), p. 647.

[2] Lin Zhou et al., Acta Materialia 164 (2019), p. 697.

[3] This work was supported in part by the U.S. Department of Energy, Office of Science, Basic Energy Sciences, Materials Sciences and Engineering Division. Laboratory Directed Research and Development funds through Ames Laboratory. All TEM-related work was performed using instruments in the Sensitive Instrument Facility in Ames Laboratory. Ames Laboratory is operated for the U.S. Department of Energy by Iowa State University under Contract No. DE-AC02-07CH11358.

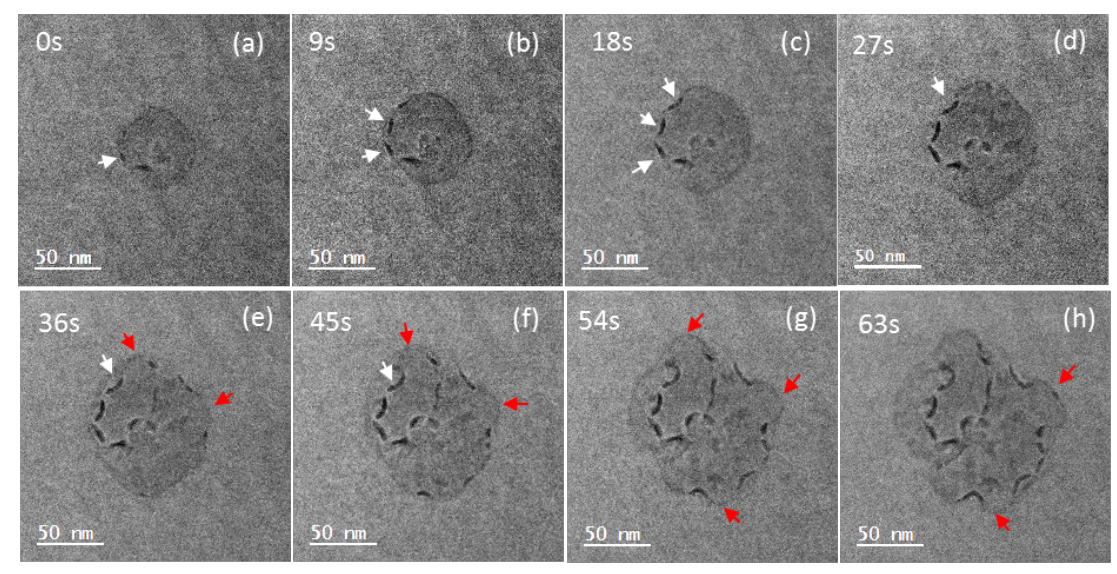

Figure 1. HAADF STEM images show the gradual growth of a $\varepsilon$ grain from the amorphous matrix. Images were taken after serially heating at $175^{\circ} \mathrm{C}$ for $9 \mathrm{~s}$. White arrows indicate position of Al-rich phase, while red arrows indicate regions which show interface piecing through two Alrich clusters.[2]

(a) $\langle 100>$

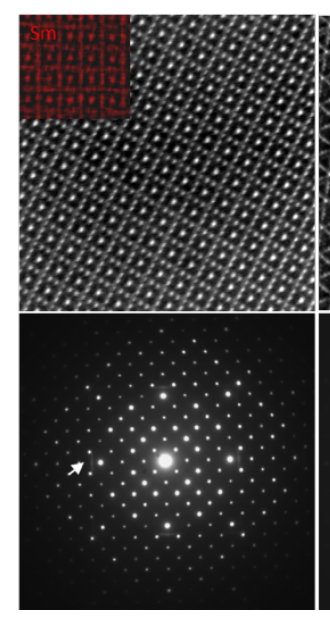

(b)

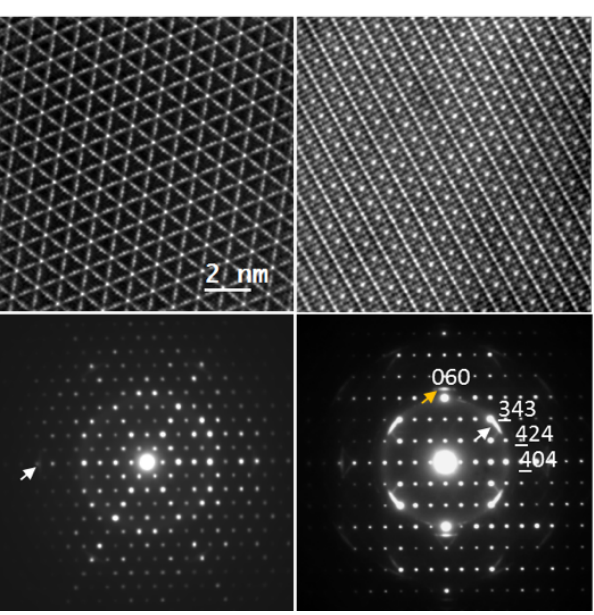

Figure 2. High resolution STEM images (top row) and corresponding diffraction pattern (bottom row) taken under (a) [100], (b) [111], and (c) [110] zone axis of the $\varepsilon$ phase. Image (a) to (c) have the same scale. Inset at top left of (a) is an EDS elemental mapping of Sm element. Arc shows in the diffraction pattern, as indicated by white arrows, are from the $f c c-\mathrm{Al}$.[2] 